\section{Rheumatoide Arthritis: Upadacitinib im Vergleich gegen Plazebo und Adalimumab}

Fleischmann R et al. Upadacitinib Versus Placebo or Adalimumab in Patients With Rheumatoid Arthritis and an Inadequate Response to Methotrexate: Results of a Phase III, DoubleBlind, Randomized Controlled Trial. Arthritis Rheumatol 2019; 71: 1788-1800

Bei Patienten mit rheumatoider Arthritis, die unzureichend auf eine Methotrexattherapie ansprachen, wurde die Wirksamkeit von Upadacitinib im Vergleich zu Plazebo oder Adalimumab getestet. Upadacitinib ist ein einmal täglich, oral einnehmbarer, selektiver JAK1-Inhibitor, der in den USA 2019 zur Behandlung der rheumatoiden Arthritis zugelassen wurde.

In eine kontrollierte, doppelblinde Phase IIIStudie wurden insgesamt 1629 Patientinnen und Patienten mit rheumatoider $\mathrm{Ar}$ thritis, die schlecht auf den Wirkstoff Methotrexat ansprachen, eingeschlossen und randomisiert entweder täglich mit $15 \mathrm{mg}$ Upadacitinib, alle 2 Wochen mit 40 mg Adalimumab oder mit Plazebo behandelt. Die Studienteilnehmer nahmen weiterhin Methotrexat ein.

Die primären Endpunkte waren das Erreichen eines ACR20-Response (American College of Rheumatology $20 \%$ ) sowie das $\mathrm{Er}$ reichen des DAS28-CRP von unter 2,6. Werte unter 2,6 entsprechen einer Remission, das heißt, der Patient hat keine Beschwerden mehr, und die Erkrankung ist zum Stillstand gekommen. Die Hemmung des radiologischen Fortschreitens der Erkrankung wurde in Woche 26 evaluiert. Die Studie war so designend und gepowert, dass die Nichtunterlegenheit und Überlegenheit von Upadacitinib im Vergleich zu Adalimumab sowohl klinisch als auch funktionell zu bestimmen war.

An der Studie beteiligten sich 286 Zentren in 41 Ländern. Der überwiegende Teil der Studienteilnehmer war weiblich(79,3\%), das Durchschnittsalter betrug $54 \pm 12$ Jahre und die Diagnose rheumatoide Arthritis bestand im Mittel seit $8 \pm 8$ Jahren.
Nach 12 Wochen erreichten $71 \%$ der Patientinnen und Patienten, die mit Upadacitinib und $36 \%$ derjenigen, die mit Plazebo behandelt wurden, eine ACR20-Response, also eine $20 \%$-Verbesserung der Gelenkbeschwerden. 29\% der Patientinnen und Patienten im Upadacitinib-Arm und $6 \%$ im Plazebo-Arm erreichten einen DAS28-CRP von unter 2,6, also einen Stillstand der Erkrankung. Im Vergleich mit Adalimumab führte Upadacitinib häufiger zu einer 50 \%igen Verbesserung der Gelenkbeschwerden. Auch die Schmerzen und die Behinderungen sowie die Krankheitsaktivität wurden stärker gesenkt als im Adalimumab-Arm.

Nach 26 Wochen kam es bei signifikant mehr Patientinnen und Patienten unter Upadacitinib als unter Plazebo und Adalimumab zu einer geringeren Krankheitsaktivität oder sogar einem Stillstand. Das im Röntgenbild sichtbare Fortschreiten der Erkrankung nahm unter der Behandlung mit Upadacitinib im Vergleich zu Plazebo deutlich ab. Die Nebenwirkungen von Upadacitinib und Adalimumab waren vergleichbar. Mehr Patientinnen und Patienten in der Adalimumab-Gruppe als in der Upadacitinib-Gruppe litten unter schweren Nebenwirkungen oder brachen die Therapie aufgrund der Nebenwirkungen ab.

Patienten unter Upadacitinib litten häufiger an Herpes zoster und einer Erhöhung der Kreatinkinase. Insgesamt kam es in der gesamten Studienpopulation zu 3 Krebserkrankungen, 5 schweren Herz-Komplikationen und 4 Todesfällen. Keine dieser Ereignisse trat im Upadacitinib-Arm auf. Es kam zu 6 Thromboembolien ( 1 in der PlazeboGruppe, 2 in der Upadacitinib-Gruppe und 3 in der Adalimumab-Gruppe).
FAZIT

Die Studienergebnisse fassen die Autoren so zusammen: Upadacitinib ist bei Patienten mit rheumatoider Arthritis, die gleichzeitig Methotrexat erhielten, besser wirksam als Plazebo und Adalimumab. Auch das Fortschreiten des Gelenkverschleißes, der im Röntgenbild zu sehen ist, konnte Upadacitinib bremsen. Die Sicherheit war mit der von Adalimumab vergleichbar. Unter Upadacitinib kam es häufiger zu Herpes zoster und einer Erhöhung der Kreatinkinase.

Richard Kessing, Zeiskam 